\title{
Limitation of Life-Sustaining Care in the Critically III: A Systematic Review of the Literature
}

\author{
Katie McPherson, MD'; W Graham Carlos III, MD²; Thomas W Emmett, MD, MLS;
} James E Slaven, MS, MA4; Alexia M Torke, MD, MS

\begin{abstract}
${ }^{1}$ Division of Pulmonary and Critical Care Medicine, University of Colorado, Denver Colorado; ${ }^{2}$ Division of Pulmonary, Critical Care, Sleep, and Occupational Medicine, Indiana University School of Medicine, Indianapolis, Indiana; ${ }^{3}$ Ruth Lilly Medical Library at Indiana University School of Medicine, Indianapolis, Indiana; ${ }^{4}$ Department of Biostatistics, Indiana University School of Medicine, Indianapolis, Indiana; Indiana University Center for Aging Research, Indianapolis Indiana; ${ }^{6}$ Daniel F. Evans Center for Spiritual and Religious Values in Healthcare and Fairbanks Center for Medical Ethics, Indiana University Health, Indianapolis, Indiana.
\end{abstract}

When life-sustaining treatments (LST) are no longer effective or consistent with patient preferences, limitations may be set so that LSTs are withdrawn or withheld from the patient. Many studies have examined the frequency of limitations of LST in intensive care unit (ICU) settings in the past 30 years. This systematic review describes variation and patient characteristics associated with limitations of LST in critically ill patients in all types of ICUs in the United States. A comprehensive search of the literature was performed by a medical librarian between December 2014 and April 2017. A total of 1,882 unique titles and abstracts were reviewed, 113 were selected for article review, and 36 studies were fully reviewed. Patient factors associated with an increased likelihood of limiting LST included white race, older age, female sex, poor preadmission functional status, multiple comorbidities, and worse illness severity score. Based on several large, multicenter studies, there was a trend toward a higher frequency of limitation of LST over time. However, there is large variability between ICUs in the proportion of patients with limitations and on the proportion of deaths preceded by a limitation. Increases in the frequency of limitations of LST over time suggests changing attitudes about aggressive end-of-life-care. Limitations are more common for patients with worse premorbid health and greater ICU illness severity. While some differences in the frequency of limitations of LST may be explained by personal factors such as race, there is unexplained wide variability between units. Journal of Hospital Medicine 2019;14:303-310. Published online first February 20, 2019. (C) 2019 Society of Hospital Medicine

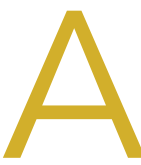

ccess to life-sustaining treatment (LST) became a mainstay in hospitals across the United States in the 1970s. This has raised complex ethical questions surrounding the use of these therapies, particularly in the face of a poor prognosis or significant morbidity. The Society for Critical Care Medicine formed a consensus panel in 1989 to construct ethical guidelines regarding the initiation, continuation, and withdrawal of intensive care. ${ }^{1}$ These guidelines emphasized that withdrawing and withholding are not only permissible but may be necessary to preserve the balance between quantity and quality of life. Nevertheless, an increasing number of Americans are dying after aggressive LST in the hospital, and greater than one in five deaths occur after admission to the ICU. ${ }^{2}$ Understanding the factors associated with decisions to withhold or withdraw LST are important to policy makers, ethicists, and healthcare leaders because they affect

\footnotetext{
*Corresponding Author: Alexia M Torke, MD, MS; E-mail: atorke@iu.edu; Telephone: 317-274-9221; Twitter: @AlexiaMTorke

Additional Supporting Information may be found in the online version of this article.
}

Received: July 7, 2018; Revised: December 3, 2018;

Accepted: December 3, 2018

(c) 2019 Society of Hospital Medicine DOI 10.12788/jhm.3137 resources used at the end of life and the need for palliative care and hospice in the ICU setting.

Several studies have characterized the patient characteristics, incidence, and variability associated with limitation of LST in various populations of critically ill patients in the US. We are unaware of another systematic review of the literature that has examined data from these studies in order to understand the process and outcomes of LST limitations. We defined limitations of LST as decisions to withdraw or withhold cardiopulmonary resuscitation through Do Not Resuscitate (DNR) orders, mechanical ventilation, renal replacement therapy, intravenous blood pressure support, or artificial nutrition (enteric or intravenous).

\section{METHODS}

The Preferred Reporting Items for Systematic Reviews and Meta-Analyses statement was used for reporting. A comprehensive literature search was performed by a medical librarian (TWE) in Ovid MEDLINE, PubMed, Embase, the full Cochrane Library, CINAHL, PsycINFO, the Philosopher's Index, Scopus, Web of Science, and Google Scholar. PubMed was limited to non-MEDLINE records in order to complement the Ovid results. The Georgetown Bioethics Research Library at the Kennedy Institute (https://bioethics.georgetown.edu/) was also searched for any unpublished literature. Initial searches were 

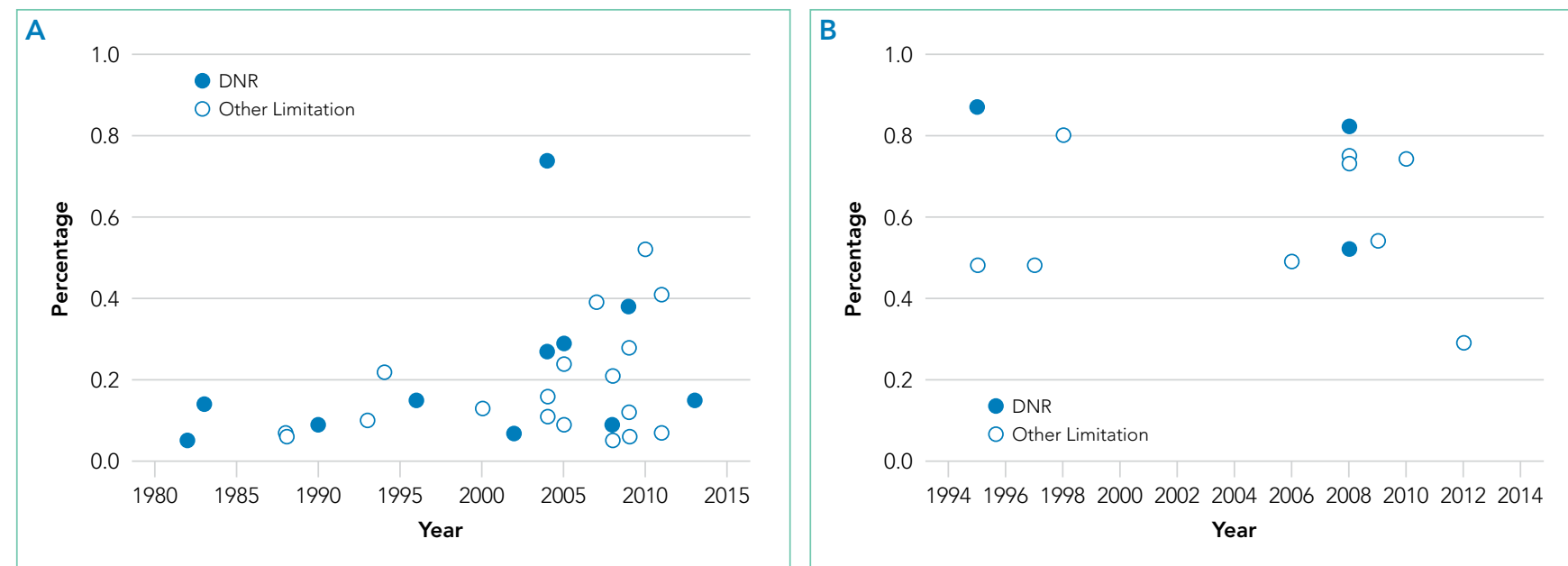

FIG 1.Percent of patients with limitations, by year, for studies that included all Intensive Care unit admissions (A) and studies that included patients who died in the ICU (B).

conducted in December 2014, and an update was performed in April 2017. All databases were searched from inception, and bibliographies of relevant studies were reviewed for additional references (Appendix 1).

Database-specific subject headings and keyword variants for each of the five main concepts-intensive care, end-of-life, decision making, limitation of treatment, and death-were identified and combined. Results were limited to the adult population and to the English language.

Two authors independently reviewed article titles and abstracts (KM, AMT). The full text of potentially eligible studies was then reviewed for inclusion. All disputes were discussed and resolved by consensus. The criteria for inclusion were reporting of patient-level data, critical care patients only (or reported separately from other unit types), US setting, and reporting of data on limitations of LST. The exclusion criteria were studies published only as research abstracts, surveys of physicians or families, organ donors, studies of brain death, surveys, patients less than 18 years old, and long-term intensive care settings (ie, long-term acute care hospitals, longterm respiratory units). Also excluded were studies in which an intervention was performed; as a result, all included studies were observational. Research abstracts were excluded because they lacked sufficient detail from which to abstract study quality or results. Studies of organ donation, brain death, and pediatrics were excluded due to differences in the decision-making context that would make it difficult to draw conclusions about adult ICU care. Studies which included an intervention were excluded to avoid affecting the rate of limitation of LST as a result of the intervention, since our goal was to quantify the number of limitations of LST in usual medical practice.

For each article, we abstracted the number of patients who experienced a limitation of LST out of the total population and factors associated with the limitation. If a multivariable analysis was performed, we reported only variables that remained significant in this analysis. We also reported the number of patients who died, and of those, the number of decedents who underwent a limitation of LST before death. In some cases, this proportion was not reported in the manuscript but could be calculated based on the data presented. This number was calculated based on the number of deaths that were preceded by a limitation in life-sustaining care divided by the total number of deaths. Patients with brain death were not counted as having had a "limitation" if support was withdrawn after the declaration of brain death. We were unable to conduct a meta-analysis of the findings because of the wide variation in study populations and criteria used to define limitations of care.

To assess risk of bias in individual studies, the two raters independently made a yes/no determination regarding several quality metrics established at the outset of the review: clarity of the eligibility criteria for participant inclusion, whether a power or sample size calculation was done, adequacy of the description of the sampling approach and recruitment, and generalizability. Disagreements were resolved by consensus.

\section{RESULTS}

\section{Study Selection}

A total of 2,460 references were identified, and after removal of 578 duplicates, 1,882 unique titles and abstracts were reviewed. One hundred thirteen titles met the inclusion criteria. After review of complete texts, 83 were excluded based on the above criteria (Appendix). This led to a final number of 36 studies included for analysis.

Fifteen articles were prospective, observational studies. The rest were retrospective analyses of patient-level data. Seven were large, multicenter studies with greater than 20 centers involved (including Project IMPACT); six such studies included medical and surgical patients. The remaining large, multicenter study examined a surgical trauma cohort.

Fifteen of the studies addressed DNR as a limitation and 25 addressed other limitations such as withdrawing or withholding LST (several addressed both DNR and another limitation). Nine studies enrolled only patients who had died and the remaining 27 enrolled all ICU admissions. 


\section{Historical Trends}

Examination of the three studies that looked at $>20$ regionally diverse ICUs revealed a trend over time toward increased limitation prior to death (Figure). Jayes looked at the number of DNR orders preceding death from 1979 to 1980 then compared that to a cohort from 1988 to 1990; Prendergast included withholding/withdrawing of LST prior to death from 1994 to 1995; and Quill used the IMPACT database to examine limitations prior to death from 2001 to 2009 . $^{3-5}$

\section{Effect of Unit Specialty}

Twelve studies were mixed (surgical/medical or medical/neuro) ICUs, 11 were medical/cardiac units, five were neurologic units, and six were surgical/trauma units only. Two studies did not report unit specialty. Four studies that compared surgical and medical ICUs found that surgical patients were more likely to die with full intervention..$^{4-7}$ In all of these studies, medical patients were more likely to have limitations of LST preceding death. Quill, et al. further detailed that emergency surgery was more likely to be associated with limitation than elective surgery. ${ }^{5}$

\section{Patient Factors}

In 15 studies, older age was associated with an increased likelihood of limitations on LST., 3-18 In one study, advanced age was associated with early versus late withdrawal. ${ }^{19}$ Poor performance status and multiple medical comorbidities were also associated with limitations of LST. The largest population-based study by Quill et al. found that being fully dependent on others upon admission to the ICU was associated with an increased likelihood of limiting LST. ${ }^{5}$ Sise et al. found, in an analysis performed over 10 years in one trauma center, that increased age, comorbidities, and a fall as the reason for trauma admission were associated with limitation of LST. ${ }^{9}$ Salottolo et al. found that if the reason for trauma admission was a fall, there was an increased odds ratio of DNR status. ${ }^{18}$ Many studies found that having medical comorbidities prior to admission was associated with increased likelihood of limiting LST in both medical and surgical patients. $3,7,9,13,15,18$

Five studies found a statistically significant difference between women and men in the likelihood of limitation of LST, 3,5,9,14,16 and another study reported that women who were trauma patients had an increased odds ratio of changing to DNR code status. ${ }^{18}$ Only one study found that males were associated with an increased likelihood of limiting aggressive treatment. ${ }^{20}$

White race was associated with increased limitation of LST in nine studies. ${ }^{4,5}, 10,11,14-16,21,22$ One study in neurocritical care patients found that both white and Hispanic races were correlated with a higher likelihood of limitations. ${ }^{23}$ Muni et al. found that nonwhite patients had a statistically significantly lower likelihood of having comfort measures and DNR orders written prior to death, but discussion of prognosis was more likely to be documented in nonwhite patients. ${ }^{21}$

In summary, white race, female gender, and older age were the most frequent factors associated with a higher likelihood of limiting LST.

\section{Factors Related to Critical IIIness}

There were several illness severity indicators that were associated with limitations. The Acute Physiology and Chronic Health Evaluation (APACHE) scores were the most common for medical patients and Glasgow Coma Scale (GCS) was the most common for patients with neurologic injury. Eight studies reported that a higher APACHE score was associated with an increased likelihood of limitations. 3,7,10,15,17,20,22,24 Similar associations were found based on the Sepsis Related Organ Failure Assessment score in one study and a scoring system developed by the author in a second study. ${ }^{25,26}$

Seven studies, consisting of three neurologic, two medical-surgical, and two trauma cohorts, reported that a lower GCS score increased the likelihood that the patient would have limited LST. $5,10,11,13,14,18,22$ Additionally, Geocadin and colleagues discussed the difficulty with neurological prognostication in clinical practice; they reported that the cortical evoked potential (CEP) was correlated with the time to withdraw LST if the CEP was malignant, and the time to withdraw LST was less in malignant than in benign CEP. ${ }^{27}$

\section{Mortality and End Effects of Limiting LST}

Chen and colleagues used propensity scores to control for mortality differences between patients who had full interventions versus those with limitations and found that higher mortality correlated with the decision to withhold or withdraw LST. ${ }^{10}$ Weimer and colleagues used modeling to predict the probable outcome of patients who experienced an intracranial hemorrhage who had limitation of LST. Based on this model, nearly all the patients in their study would have died or had severe disability at 12 months despite having maximal therapy; they concluded that withdrawal of LST may not have been a self-fulfilling prophecy as others have proposed. ${ }^{28}$ Mulder and colleagues reported that in a small cohort of out-of-hospital cardiac arrest survivors admitted to the hospital, over one-third had good neurological outcomes after coding after 72 hours. ${ }^{29}$ The study highlighted the importance of timing in neurological prognostication.

\section{Variation in Limitation Rates among Centers}

In the 36 studies, we found an overall range of DNR orders from $5.4 \%^{7}$ to $82.0 \% .^{30}$ For other limitations, the rates ranged from $6.3 \%{ }^{13}$ to $80.4 \% .{ }^{31}$ Hart reported a low rate of limitations (4.8\%) at the time of ICU admission. ${ }^{16}$ Four large, multicenter studies drew attention to the large variability between critical care centers and the limitation of end-of-life care..$^{3-5,14}$ Jayes first described this phenomenon when examining the frequency of DNR orders from 1979 to 1980 and 1988 to $1990 .^{3}$ This study found a range from $1.5 \%$ to $22 \%$. Later, in another large, multicenter study, Prendergast et al. looked at 131 ICUs at 110 different institutions in 38 states that participated in postgraduate training and found variability in CPR attempts prior to death between $4 \%$ and 79\%. ${ }^{4}$ In 2008, Nathens et al. reported significant variation in DNR rates across trauma centers; they found a higher incidence of DNR orders when there was an open ICU structure. $^{14}$ 
TABLE. Main Results of the 36 Final Manuscripts

\begin{tabular}{|c|c|c|c|c|c|c|c|c|}
\hline Article Year & $\begin{array}{c}\text { Period of } \\
\text { Data Collection }\end{array}$ & $\begin{array}{l}\text { Study Population } \\
\text { (Number, } \\
\text { Description, Study) }\end{array}$ & $\begin{array}{l}\text { Limitation } \\
\text { Addressed } \\
\text { in the Study }\end{array}$ & $\begin{array}{l}\text { Type of Intensive } \\
\text { Care Unit }\end{array}$ & Study Design & $\begin{array}{l}\text { Number (\%) with } \\
\text { Limitation of LST }\end{array}$ & $\begin{array}{l}\text { Of Those Who Died, } \\
\text { What Number (\%) } \\
\text { Had Limitations }\end{array}$ & $\begin{array}{c}\text { Patient } \\
\text { Characteristics } \\
\text { Associated with } \\
\text { Limitation }\end{array}$ \\
\hline Albaeni $2014^{19}$ & $2004-2010$ & $\begin{array}{l}189 \text { survivors of out-of- } \\
\text { hospital cardiac arrest } \\
\text { pts; } 1 \text { unit }\end{array}$ & Withdrawal of LST & Cardiac/medical & Retrospective & $99 / 189(52 \%)$ & $\begin{array}{c}\text { In hospital death 94/147 } \\
(63.9 \%)\end{array}$ & $\begin{array}{l}\text { No associations } \\
\text { were found }\end{array}$ \\
\hline Brown $2016^{30}$ & $2003-2008$ & $\begin{array}{l}829 \text { pts who died in } \\
\text { ICUs from ILD, COPD, or } \\
\text { metastatic; } 15 \text { hospitals, } \\
2 \text { neuro ICUs, and } 5 \\
\text { non-neuro ICUs }\end{array}$ & $\begin{array}{c}\text { DNR, withdrawal } \\
\text { of LST }\end{array}$ & Medical & $\begin{array}{l}\text { Secondary analysis } \\
\text { of randomized trial }\end{array}$ & $\begin{array}{c}\text { DNR 681/829 (82.1\%); } \\
\text { Withdraw LST 621/829 } \\
(74.9 \%)\end{array}$ & $\begin{array}{c}\text { DNR 681/829 (82.1\%); } \\
\text { Withdraw LST 621/829 } \\
(74.9 \%)\end{array}$ & Not reported \\
\hline Chen $2008^{10}$ & $2002-2005$ & $\begin{array}{l}\text { 2,211 consecutive ICU } \\
\text { admissions; } 1 \text { unit }\end{array}$ & $\begin{array}{l}\text { Withholding LST, } \\
\text { excluded withdraw } \\
\text { of treatment }\end{array}$ & Medical & Retrospective & $201 / 2,211(9.1 \%)$ & Not reported & $\begin{array}{c}\text { Age, GCS, APACHE } \\
\text { II, race }\end{array}$ \\
\hline $\begin{array}{l}\text { Creutzfeldt } \\
2015^{36}\end{array}$ & $2001-2008$ & $\begin{array}{l}200 \text { admission to } \\
2 \text { neuro ICUs who met } \\
\text { palliative care triggers, } \\
1,909 \text { admissions to } \\
5 \text { other ICUs in the } \\
\text { same hospitals } \\
\text { who met triggers } \\
\text { (Project IMPACT) }\end{array}$ & $\begin{array}{l}\text { DNR, withholding } \\
\text { LST }\end{array}$ & Neuro vs other & Retrospective & $\begin{array}{c}\text { Neuro ICU DNR 16/175 } \\
(9.1 \%) ; \text { WD/WH } 36 / 175 \\
(20.6 \%) ; \\
\text { Non-neuro ICU DNR } \\
\text { 212/1,711 (12.4\%); } \\
\text { WD/WH 195/1,711 } \\
(11.4 \%)\end{array}$ & Not reported & Not reported \\
\hline Diringer $2001^{11}$ & 1994- 2000 & $\begin{array}{c}2,109 \text { pts treated with } \\
\text { mechanical ventilation; } \\
1 \text { unit }\end{array}$ & $\begin{array}{l}\text { Withdrawal } \\
\text { of mechanical } \\
\text { ventilation }\end{array}$ & $\begin{array}{l}\text { Neurology/ } \\
\text { Neurosurgery }\end{array}$ & $\begin{array}{c}\text { Retrospective analysis } \\
\text { of prospectively } \\
\text { collected clinical } \\
\text { database }\end{array}$ & $284 / 2,109(13.5 \%)$ & $279 / 720(38.8 \%)$ & $\begin{array}{l}\text { Surgical pts, age, } \\
\text { GCS, race }\end{array}$ \\
\hline Geocadin $2006^{27}$ & Dates not given & $\begin{array}{l}58 \text { comatose pts after } \\
\text { cardiac arrest consulted } \\
\text { on by the neurology } \\
\text { service; } 1 \text { unit }\end{array}$ & $\begin{array}{l}\text { Withholding and } \\
\text { withdrawal of LST }\end{array}$ & Cardiac, medical & $\begin{array}{c}\text { Prospective } \\
\text { observational cohort }\end{array}$ & 40/58 (70.0\%) & 40/48 (83.3\%) & Not reported \\
\hline Hamel $2002^{25}$ & 1989- 1994 & $\begin{array}{c}596 \text { Non-traumatic } \\
\text { coma pts (SUPPORT); } 5 \\
\text { medical centers }\end{array}$ & $\begin{array}{l}\text { Withholding CPR } \\
\text { and ventilation }\end{array}$ & Unknown & $\begin{array}{l}\text { Secondary analysis } \\
\text { of prospective study }\end{array}$ & $121 / 549(22.0 \%)$ & Not reported & $\begin{array}{c}\text { Higher risk } \\
\text { assessment score }\end{array}$ \\
\hline Hart $2015^{16}$ & $2001-2008$ & $\begin{array}{l}\text { 277,693 ICU admissions } \\
\text { (Project IMPACT ); } 141 \\
\text { ICUs in } 105 \text { hospitals }\end{array}$ & $\begin{array}{l}\text { Treatment limitation } \\
\text { at the time of ICU } \\
\text { admission }\end{array}$ & Mixed & Retrospective & $13,405 / 277,693(4.8 \%)$ & Not reported & $\begin{array}{l}\text { Age, gender, race, } \\
\text { pre-admission } \\
\text { functional status }\end{array}$ \\
\hline Huynh $2013^{12}$ & 2005- 2006 & $\begin{array}{l}322 \text { ICU pts who died; } \\
1 \text { hospital }\end{array}$ & $\begin{array}{l}\text { Withdrawal } \\
\text { of mechanical } \\
\text { ventilation }\end{array}$ & Not specified & Retrospective & 159/322 (49.4\%) & 159/322 (49.4\%) & Age, nonsurgical pts \\
\hline Jayes $1993^{3}$ & $1982 ; 1988-1990$ & $\begin{array}{l}\text { 7,265 ICU admissions } \\
\text { 1979-1982 17,440 ICU } \\
\text { admissions from } \\
1988 \text {-1990; } 42 \text { ICUs }\end{array}$ & DNR & Medical, surgical & $\begin{array}{l}\text { Prospective inception } \\
\text { cohort }\end{array}$ & $\begin{array}{c}1979-1982393 / 7,265 \\
(5.4 \%) \\
1988-1990 \\
1,577 / 17,440(9.0 \%)\end{array}$ & $\begin{array}{l}60 \% \text { in } 1988-1990 ; \\
39 \% \text { in } 1979-1982\end{array}$ & $\begin{array}{c}\text { Age, sex, APACHE III } \\
\text { score, pre-admission } \\
\text { daily living activities, } \\
\text { chronic disease }\end{array}$ \\
\hline Kerlin $2015^{43}$ & $2001-2008$ & $\begin{array}{c}\text { 270,742 ICU } \\
\text { admissions (Project } \\
\text { IMPACT); } 143 \text { ICUs }\end{array}$ & Limitation of LST & Mixed & Retrospective & Not reported & Not reported & Not reported \\
\hline Kish Wallace ${ }^{44}$ & 1994-1996 & $\begin{array}{c}270 \text { admissions to ICU; } \\
1 \text { hospital }\end{array}$ & DNR & $\begin{array}{l}\text { Medical ICU in } \\
\text { cancer hospital }\end{array}$ & $\begin{array}{l}\text { Matched pairs case } \\
\text { control }\end{array}$ & $41 / 270(15.2 \%)$ & Unable to determine & Matched groups \\
\hline Kowalski $2013^{13}$ & $1991-2009$ & $\begin{array}{l}\text { 1,134 aneurysmal SAH } \\
\text { pts; } 2 \text { units }\end{array}$ & Withdrawal of LST & $\begin{array}{l}\text { Neurology/ } \\
\text { neurosurgery }\end{array}$ & $\begin{array}{l}\text { Prospective } \\
\text { observational }\end{array}$ & $72 / 1,134(6.3 \%)$ & $\begin{array}{l}\text { In hospital deaths } \\
72 / 207(34.7 \%)\end{array}$ & $\begin{array}{l}\text { GCS, age, } \\
\text { comorbidities }\end{array}$ \\
\hline Lissauer $2011^{20}$ & 2008- 2010 & $\begin{array}{l}151 \text { surgical ICU pts } \\
\text { who died; } 1 \text { unit }\end{array}$ & $\begin{array}{l}\text { Withdrawal and } \\
\text { withholding of LST }\end{array}$ & Surgical & $\begin{array}{l}\text { Retrospective analysis } \\
\text { of prospectively } \\
\text { collected data }\end{array}$ & $111 / 151(73.5 \%)$ & $111 / 151(73.5 \%)$ & Sex, APACHE IV score \\
\hline
\end{tabular}


TABLE. Main Results of the 36 Final Manuscripts (continued)

\begin{tabular}{|c|c|c|c|c|c|c|c|c|}
\hline Article Year & $\begin{array}{c}\text { Period of } \\
\text { Data Collection }\end{array}$ & $\begin{array}{l}\text { Study Population } \\
\text { (Number, } \\
\text { Description, Study) }\end{array}$ & $\begin{array}{l}\text { Limitation } \\
\text { Addressed } \\
\text { in the Study }\end{array}$ & $\begin{array}{l}\text { Type of Intensive } \\
\text { Care Unit }\end{array}$ & Study Design & $\begin{array}{l}\text { Number (\%) with } \\
\text { Limitation of LST }\end{array}$ & $\begin{array}{c}\text { Of Those Who Died, } \\
\text { What Number (\%) } \\
\text { Had Limitations }\end{array}$ & $\begin{array}{c}\text { Patient } \\
\text { Characteristics } \\
\text { Associated with } \\
\text { Limitation }\end{array}$ \\
\hline Mayer $1999^{23}$ & 1994- 1997 & $\begin{array}{l}105 \text { neurocritical ICU } \\
\text { pts who died; } 1 \text { unit }\end{array}$ & $\begin{array}{l}\text { Withdrawal of } \\
\text { ventilation }\end{array}$ & Neuro & Retrospective & 50/105 (47.6\%) & 50/105 (47.6\%) & Race \\
\hline Mehter $2014^{17}$ & $2000-2005$ & $\begin{array}{l}809 \text { ICU pts with ARDS } \\
\text { and documented code } \\
\text { status; multicenter }\end{array}$ & $\begin{array}{l}\text { DNR, withdrawal } \\
\text { and withholding } \\
\text { of LST }\end{array}$ & Medical & $\begin{array}{l}\text { Retrospective } \\
\text { observation of clinical } \\
\text { trial subset }\end{array}$ & $\begin{array}{c}\text { DNR 232/809 }(28.7 \%) \\
\text { WD/WH 195/809 } \\
(24.1 \%)\end{array}$ & $\begin{array}{l}90 \text { day mortality DNR } \\
225 / 284(79.2 \%)\end{array}$ & APACHE III score \\
\hline $\begin{array}{l}\text { Mulder } 2013^{37} \\
\text { and } 2014^{29}\end{array}$ & $2007-2011$ & $\begin{array}{l}154 \text { comatose survivors } \\
\text { out of hospital cardiac } \\
\text { arrest survivors } \\
\text { admitted to the ICU; } \\
1 \text { center }\end{array}$ & Withdrawal of LST & Medical & $\begin{array}{l}\text { Prospective } \\
\text { observational }\end{array}$ & $63 / 154(40.9 \%)$ & $63 / 78(80.8 \%)$ & Not reported \\
\hline Muni $2011^{21}$ & 2003- 2008 & $\begin{array}{c}\text { 3,138 ICU pts } \\
\text { who died; } 15 \text { ICUs }\end{array}$ & $\begin{array}{l}\text { DNR, comfort care, } \\
\text { RRT, MV, pressors, } \\
\text { enteral feedings }\end{array}$ & Medical & Retrospective & $2,274 / 3,121(72.90 \%)$ & $2,274 / 3,121(72.90 \%)$ & Race \\
\hline Naib $2015^{38}$ & 2012 & 1,368 pts; 1 CICU & $\begin{array}{l}\text { Withdrawal and } \\
\text { withholding of LST }\end{array}$ & Cardiac & Retrospective & Not reported & $\begin{array}{c}\text { ICU deaths } 85 / 117 \\
(72.6 \%)\end{array}$ & Not reported \\
\hline Nathens $2008^{14}$ & 2001- 2002 & $\begin{array}{l}6,765 \text { trauma pts; } 68 \\
\text { centers }\end{array}$ & DNR & Trauma, mixed & $\begin{array}{l}\text { Prospective } \\
\text { observational }\end{array}$ & $464 / 6,765(6.9 \%)$ & $408 / 60367.7 \%$ & $\begin{array}{l}\text { Age, race, sex, global } \\
\text { injury severity score, } \\
\text { admission GCS }\end{array}$ \\
\hline Plaisier $2002^{31}$ & 1994- 1998 & $\begin{array}{l}102 \text { trauma pts who } \\
\text { died; } 1 \text { unit }\end{array}$ & $\begin{array}{l}\text { Withdrawal and } \\
\text { withholding of LST }\end{array}$ & Trauma & Retrospective & $82 / 102(80.4 \%)$ & $82 / 102(80.4 \%)$ & Not reported \\
\hline $\begin{array}{l}\text { Prendergast } \\
1997^{39}\end{array}$ & $\begin{array}{l}\text { 1987-1988;1992- } \\
1993\end{array}$ & $\begin{array}{l}\text { 1987-1988: } 1,719 \text { ICU } \\
\text { admissions } \\
\text { 1992-1993: 1,711 ICU } \\
\text { admissions; } 2 \text { hospitals }\end{array}$ & $\begin{array}{l}\text { Withdrawal and } \\
\text { withholding of LST }\end{array}$ & Medical/surgical & $\begin{array}{l}\text { Retrospective } \\
\text { for 1987-1988, } \\
\text { prospective for 92-93 }\end{array}$ & $\begin{array}{c}1987-1988114 / 1719 \\
(6.6 \%) ; 1992-1993 \\
177 / 1711(10 \%)\end{array}$ & $\begin{array}{c}1987-1988114 / 224 \\
(50.9 \%) ; 1992-1993 \\
179 / 200(89.5 \%)\end{array}$ & Not reported \\
\hline $\begin{array}{l}\text { Prendergast } \\
1998^{4}\end{array}$ & 1994- 1995 & $\begin{array}{c}\text { 6,303 ICU pts who died; } \\
131 \text { ICUs }\end{array}$ & $\begin{array}{l}\text { DNR, withdrawal } \\
\text { and withholding } \\
\text { of LST }\end{array}$ & Medical/surgical & $\begin{array}{l}\text { Prospective } \\
\text { observational }\end{array}$ & $\begin{array}{c}\text { DNR } 5,506 / 6,303 \\
75.5 \% ; \text {; LST 3,036/6,303 } \\
\text { (48.2\%) }\end{array}$ & $\begin{array}{c}\text { DNR 5,506/6,303 } \\
\text { 75.5\%; LST 3,036/6,303 } \\
(48.2 \%)\end{array}$ & Not reported \\
\hline Quill $2014^{5}$ & 2001- 2009 & $\begin{array}{l}\text { 269,002 full code ICU } \\
\text { admissions; } 153 \text { units }\end{array}$ & $\begin{array}{l}\text { Withdrawal and } \\
\text { withholding of LST }\end{array}$ & Medical/surgical & Retrospective & $\begin{array}{c}31,408 / 269,002 \\
(11.7 \%)\end{array}$ & $\begin{array}{c}\text { ICU deaths } \\
18,460 / 21,758(84.8 \%) ; \\
\text { Hospital deaths } \\
23,469 / 33,910(69.2 \%)\end{array}$ & $\begin{array}{c}\text { Surgery type, age, } \\
\text {; race, sex, GCS score, } \\
\text { dependent pre- } \\
\text { admission status }\end{array}$ \\
\hline Reichner $2006^{26}$ & 2002- 2004 & $\begin{array}{l}47 \text { lung cancer pts } \\
\text { admitted to ICU/1 unit }\end{array}$ & $\begin{array}{l}\text { DNR/terminal } \\
\text { extubation }\end{array}$ & Medical & Retrospective & $\begin{array}{c}\text { DNR on ICU admission } \\
12 / 47(25.5 \%) ; \text { any DNR } \\
\text { order } 35 / 47(74.5 \%) ; \\
\text { terminally extubated } \\
5 / 47(10.6 \%)\end{array}$ & Not reported & Sorse SOFA score \\
\hline Rubin $2014^{22}$ & 2002- 2009 & $\begin{array}{c}1885 \text { pts with } \\
\text { neurologic injury } \\
\text { with a GCS }<9 \text {, and } \\
\text { mechanical ventilation; } \\
1 \text { unit }\end{array}$ & $\begin{array}{l}\text { Withdrawal of } \\
\text { ventilation, DNR }\end{array}$ & Neuro & $\begin{array}{l}\text { Prospective } \\
\text { observational }\end{array}$ & $\begin{array}{c}529 / 1,885(28 \%) \\
\text { withdrawal ventilation; } \\
714 / 1,885(37.9 \%) \text { DNR }\end{array}$ & $533 / 788(67.6 \%)$ & $\begin{array}{l}\text { Nonsurgical pts low } \\
\text { GCS, high APACHE II } \\
\text { white race }\end{array}$ \\
\hline Salottolo $2015^{18}$ & 2008- 2013 & $\begin{array}{l}\text { 10,053 trauma pts; } \\
\quad 1 \mathrm{ICU}\end{array}$ & $\begin{array}{l}\text { DNR, withdrawal of } \\
\text { LST noted for deaths } \\
\text { only }\end{array}$ & Surgical trauma & Retrospective & $1,523(15.1 \%)$ & $\begin{array}{c}\text { In hospital death or } \\
\text { hospice discharge, DNR } \\
336 / 455(73.8 \%) ; \\
\text { withdraw LST 45/455 } \\
(9.9 \%)\end{array}$ & $\begin{array}{l}\text { Age, sex, GCS score, } \\
\text { Charlson Comorbidity } \\
\text { Index, fall/head injury }\end{array}$ \\
\hline Sise $2012^{9}$ & 2000-2009 & $\begin{array}{l}698 \text { trauma pts who } \\
\text { died; } 1 \text { unit }\end{array}$ & $\begin{array}{l}\text { Withdrawal or } \\
\text { withholding of LST }\end{array}$ & Trauma & Retrospective & $375 / 698(53.7 \%)$ & $375 / 698(53.7 \%)$ & $\begin{array}{l}\text { Age, sex, ISS score, } \\
\text { GCS score, fall, } \\
\text { Charlson Comorbidity } \\
\text { Index, trauma consult }\end{array}$ \\
\hline Smedira $1990^{40}$ & 1987- 1988 & $\begin{array}{l}\text { 1,719 ICU patient; } 2 \\
\text { hospitals }\end{array}$ & $\begin{array}{l}\text { Withdrawal or } \\
\text { withholding of LST }\end{array}$ & Medical/surgical & $\begin{array}{l}\text { Prospective } \\
\text { observational }\end{array}$ & $97 / 1,719^{a}(5.6 \%)$ & $\begin{array}{c}\text { ICU deaths 71/109pts } \\
(62.1 \%)\end{array}$ & Not reported \\
\hline
\end{tabular}


TABLE. Main Results of the 36 Final Manuscripts (continued)

\begin{tabular}{|c|c|c|c|c|c|c|c|c|}
\hline Article Year & $\begin{array}{c}\text { Period of } \\
\text { Data Collection }\end{array}$ & $\begin{array}{l}\text { Study Population } \\
\text { (Number, } \\
\text { Description, Study) }\end{array}$ & $\begin{array}{l}\text { Limitation } \\
\text { Addressed } \\
\text { in the Study }\end{array}$ & $\begin{array}{l}\text { Type of Intensive } \\
\text { Care Unit }\end{array}$ & Study Design & $\begin{array}{l}\text { Number (\%) with } \\
\text { Limitation of LST }\end{array}$ & $\begin{array}{l}\text { Of Those Who Died, } \\
\text { What Number (\%) } \\
\text { Had Limitations }\end{array}$ & $\begin{array}{c}\text { Patient } \\
\text { Characteristics } \\
\text { Associated with } \\
\text { Limitation }\end{array}$ \\
\hline Turnbull $2014^{6}$ & 2004- 2007 & $\begin{array}{l}490 \text { acute lung injury } \\
\text { patient; } 13 \text { ICUs }\end{array}$ & $\begin{array}{l}\text { Withdrawal or } \\
\text { withholding of LST }\end{array}$ & Medical/surgical & $\begin{array}{l}\text { Prospective } \\
\text { observational }\end{array}$ & $192 / 490(39.2 \%)$ & $\begin{array}{l}\text { ICU deaths } 166 / 214 \\
(77.60 \%)\end{array}$ & Patient type, age \\
\hline Van Scoy $2013^{41}$ & 2006- 2008 & $\begin{array}{c}100 \text { pts who died after } \\
72 \text { hour stay in the ICU; } \\
1 \text { hospital }\end{array}$ & DNR & Medical/surgical & Retrospective & $52 / 100(52.0 \%)$ & $52 / 100(52.0 \%)$ & APACHE II score \\
\hline Weimer $2016^{28}$ & 2008- 2011 & $\begin{array}{c}383 \text { intracranial } \\
\text { hemorrhage pts; } 1 \text { ICU }\end{array}$ & Withdrawal of LST & Neuro & $\begin{array}{l}\text { Retrospective analysis } \\
\text { of prospectively } \\
\text { collected data }\end{array}$ & $26 / 383(6.8 \%)$ & (26/67) $38.8 \%$ & None \\
\hline Weireter $2014^{8}$ & 2008- 2012 & $\begin{array}{l}536 \text { trauma pts who } \\
\text { died; } 1 \text { unit }\end{array}$ & Withdrawal of LST & Trauma & $\begin{array}{l}\text { Retrospective analysis } \\
\text { of prospectively } \\
\text { collected data }\end{array}$ & $158 / 536(29.5 \%)$ & $158 / 536(29.5 \%)$ & Older age \\
\hline White $2006^{42}$ & $2003-2004$ & 303 ICU pts; 1 hospital & $\begin{array}{l}\text { DNR, limitation } \\
\text { of LST }\end{array}$ & Medical & $\begin{array}{l}\text { Prospective } \\
\text { observational }\end{array}$ & $\begin{array}{c}\text { DNR } 13 / 49(26.5 \%) \\
\text { Withdrawal LST } 8 / 49 \\
(16.3 \%)\end{array}$ & $\begin{array}{c}\text { DNR 9/13 }(69.2 \%)_{i} \\
\text { Withdrawal of LST 8/13 } \\
(61.5 \%)\end{array}$ & \\
\hline Youngner $1985^{15}$ & $1983-1983$ & $\begin{array}{c}506 \text { MICU admissions; } \\
1 \text { unit }\end{array}$ & DNR & Medical & $\begin{array}{l}\text { Prospective } \\
\text { observational }\end{array}$ & $71 / 506(14 \%)$ & $\begin{array}{l}\text { Hospital deaths } 62 / 123 \\
(50.4 \%)\end{array}$ & $\begin{array}{l}\text { Age, race, APACHE } \\
\text { score, pre-admission } \\
\text { health status }\end{array}$ \\
\hline $\begin{array}{l}\text { Zimmerman } \\
1986^{7}\end{array}$ & 1979-1982 & $\begin{array}{c}7265 \text { ICU admissions; } \\
13 \text { units }\end{array}$ & DNR & Mixed & $\begin{array}{l}\text { Prospective } \\
\text { observational }\end{array}$ & $393 / 7,265(5.4 \%)$ & $\begin{array}{c}\text { ICU deaths } 237 / 611 \\
(39 \%)\end{array}$ & $\begin{array}{l}\text { Surgery type, age, } \\
\text { APACHE score, } \\
\text { pre-admission health } \\
\text { status }\end{array}$ \\
\hline
\end{tabular}

aPts who were brain dead were not counted as having life-sustaining treatments withdrawn or withheld.

Abbreviations: APACHE, acute physiology and chronic health evaluation; COPD: chronic obstructive pulmonary disease; DNR, do not resuscitate; GCS, Glasgow coma scale ICU, intensive care unit; IMPACT, informing the pathway of COPD treatment; LST, life-sustaining treatments; MV, mechanical ventilation; Pts, patients; RRT, rapid response team; SAH, subarachnoid hemorrhage; SUPPORT: study to understand prognoses and preferences for outcomes and risks of treatments; WD, withdraw; WH, withhold.

Overall, there was wide variation in the proportion of deaths preceded by limitation of LST, ranging from $29.5 \%$ in one study of trauma patients ${ }^{8}$ to $92 \%$ in another study of trauma patients whose death occurred after 24 hours of care. ${ }^{9}$ In the largest study to date by Quill and colleagues utilizing the IMPACT database, they found large variability in the number of deaths preceded by full intervention based on differences in practice patterns of critical care centers. ${ }^{5}$

\section{Bias}

All studies indicated clear eligibility criteria for inclusion and described their sampling approach in adequate detail. All but one stated their method of participant recruitment, and the one remaining study was a secondary analysis and referenced the earlier manuscript. ${ }^{30}$ No study provided a power or sample size calculation, and sample sizes varied widely. Generalizability was most affected by the fact that many studies were conducted in a single ICU.

\section{DISCUSSION}

This systematic review of LST in US ICUs found several patient and illness factors that were associated with limitation of LST. The association of preadmission functional status and comorbidities with limitation of LST suggest that prior health is a factor in decision making. Further, ICU severity of illness, as measured by several commonly used indices, was associated with limitations.
Although variations in study design precluded meta-analysis, examination of the largest studies suggests that limitations are becoming more frequent over time. Also, early studies generally addressed DNR status, while later studies included withdrawal or withholding of LST, most commonly artificial ventilation. These findings reflect the current consensus in US medicine that it is ethically acceptable to limit LSTs in cases when they no longer benefit the patient or the patient would no longer want them. ${ }^{32,33}$

Some studies found variability by unit type, suggesting that decision making may differ among surgical, medical, and neurologic illness. Mayer and Kossoff concluded, in study of a cohort of neurocritical care ICU patients, that medical patients often have issues of physiologic futility and imminent death, whereas neurologic patients more often confront issues of quality of life. They also note that there is a difference in how patients with differing illnesses die; medical patients will have limitation of hemodialysis or vasopressors, whereas neurologic surrogate decision makers often confront decisions around terminal extubation. ${ }^{23}$

Some patient-level factors, such as race or ethnicity, may point to cultural differences in preferences for LST at the end of life. Other authors have documented that African American patients are more likely to choose end-of-life care for themselves or their family members, which may be due to cultural or religious factors as well as to a history of unequal access to 
medical care. ${ }^{34}$ Reasons for the finding that women are more likely to have limitations has not been as well described. Further research could explore whether this is due to differences in patient preferences by gender or to other factors.

Even when examining patient-level factors, illness severity and type of ICU, the wide variability in end-of-life care in critical care units across the country is still large. A worldwide review also found a high degree of variability, even within geographical regions. ${ }^{35}$ More research is needed to understand the factors associated with this wide variability, as this seems to indicate that approaches to end-of-life care may vary based on the ICU as much as individual patient preferences or clinical factors.

These findings can inform clinicians about variables that are important in the decision-making process. Patient age and race are factors to consider in the likelihood of reaching a decision to set limitations. Information about patients' health status prior to critical illness, as well as ICU illness severity, are also important considerations.

The limitations of this review include the wide variety of LSTs assessed, including code status change, ventilator withdrawal, removal of pressors, and cessation of renal replacement therapy. Also, there was variation in sample size and the number of included units. There was also significant heterogeneity in the outcomes addressed and the variety of methods used in the included studies. We attempted to address this with an analysis of the quality of the studies, but given the wide variability, we were unable to account for all of the differences; unfortunately, this is a standard issue within studies that utilize systematic reviews, as well as similar concepts such as meta-analyses.

In conclusion, the increase in the frequency of limitations of LST in critically ill patients and a change in the nature of limitations from DNR order to withdrawal or withholding of LST suggests a trend toward growing acceptance of limiting treatments in critical illness. The wide variation in withdrawal of care in US ICUs does not seem fully explained by patient variables including preferences, illness type, or changes over time. Factors such as poor prefunctional status, a higher number of comorbid conditions prior to critical illness, and the severity of critical illness are likely important for surrogates and clinicians to consider during goals of care discussions. Further research is needed to explore why patients may receive very different types of care at the end of life depending the institution and ICU in which they receive their care.

Disclosures: The authors have no conflicts of interest to disclose. This work was performed at the Indiana University School of Medicine.

Funding: Financial support for Dr. Torke was provided by a Midcareer Investigator Award in Patient Oriented Research from the National Institute on Aging (K24AG053794). Dr. McPherson was supported by the Indiana University Department of Medicine.

\section{References}

1. Sprung $\mathrm{CL}$, Raphaely RC, Hynninen M, et al. Consensus report on the ethics of foregoing life-sustaining treatments in the critically ill. Task Force on Ethics of the Society of Critical Care Medicine. Crit Care Med. 1990;18(12):1435-
1439.

2. Angus DC, Barnato $A E$, Linde-Zwirble WT, et al. Use of intensive care at the end of life in the United States: an epidemiologic study. Crit Care Med. 2004;32(3):638-643.

3. Jayes RL, Zimmerman JE, Wagner DP, Draper EA, Knaus WA. Do-not-resuscitate orders in intensive care units. Current practices and recent changes. JAMA. 1993;270(18):2213-2217. doi: 10.1001/jama.1993.03510180083039.

4. Prendergast TJ, Claessens MT, Luce JM. A national survey of end-of-life care for critically ill patients. Am J Respir Crit Care Med. 1998;158(4):1163-1167. doi: 10.1164/ajrccm.158.4.9801108.

5. Quill CM, Ratcliffe SJ, Harhay MO, Halpern SD. Variation in decisions to forgo life-sustaining therapies in US ICUs. Chest. 2014;146(3):573-582. doi: 10.1378/chest.13-2529.

6. Turnbull AE, Ruhl AP, Lau BM, Mendez-Tellez PA, Shanholtz CB, Needham DM. Timing of limitations in life support in acute lung injury patients: a multisite study. Crit Care Med. 2014;42(2):296-302. doi: 10.1097/ CCM.0b013e3182a272db.

7. Zimmerman JE, Knaus WA, Sharpe SM, Anderson AS, Draper EA, Wagner DP. The use and implications of do not resuscitate orders in intensive care units. JAMA. 1986;255(3):351-356. doi: 10.1001/jama.1986.03370030071030.

8. Weireter LJ, Jr., Collins JN, Britt RC, Novosel TJ, Britt LD. Withdrawal of care in a trauma intensive care unit: the impact on mortality rate. Am Surg. 2014;80(8):764-767.

9. Sise MJ, Sise CB, Thorndike JF, Kahl JE, Calvo RY, Shackford SR. Withdrawal of care: A 10-year perspective at a Level I trauma center. J Trauma Acute Care Surg. 2012;72(5):1186-1191. doi: 10.1097/TA.0b013e31824d0e57.

10. Chen Y-Y, Connors AF, Jr., Garland A. Effect of decisions to withhold life support on prolonged survival. Chest. 2008;133(6):1312-1318. doi: 10.1378/ chest.07-1500.

11. Diringer MN, Edwards DF, Aiyagari V, Hollingsworth $\mathrm{H}$. Factors associated with withdrawal of mechanical ventilation in a neurology/neurosurgery intensive care unit. Crit Care Med. 2001;29(9):1792-1797.

12. Huynh TN, Walling AM, Le TX, Kleerup EC, Liu H, Wenger NS. Factors associated with palliative withdrawal of mechanical ventilation and time to death after withdrawal. J Palliat Med. 2013;16(11):1368-1374. doi: 10.1089/ jpm.2013.0142.

13. Kowalski RG, Chang TR, Carhuapoma JR, Tamargo RJ, Naval NS. Withdrawal of technological life support following subarachnoid hemorrhage. Neurocrit Care. 2013;19:269-275. doi: 10.1007/s12028-013-9929-8.

14. Nathens AB, Rivara FP, Wang J, Mackenzie EJ, Jurkovich GJ. Variation in the rates of do not resuscitate orders after major trauma and the impact of intensive care unit environment. J Trauma. 2008;64(1):81-88; discussion 8-91. doi: 10.1097/TA.0b013e31815dd4d7.

15. Youngner SJ, Lewandowski W, McClish DK, Juknialis BW, Coulton C, Bartlett ET. 'Do not resuscitate' orders. Incidence and implications in a medical-intensive care unit. JAMA. 1985;253(1):54-57. doi: 10.1001/ jama.1985.03350250062023.

16. Hart JL, Harhay MO, Gabler NB, Ratcliffe SJ, Quill CM, Halpern SD. Variability among US intensive care units in managing the care of patients admitted with preexisting limits on life-sustaining therapies. JAMA Intern Med. 2015;175(6):1019-1026. doi: 10.1001/jamainternmed.2015.0372.

17. Mehter HM, Wiener RS, Walkey AJ. "Do not resuscitate" decisions in acute respiratory distress syndrome: a secondary analysis of clinical trial data. Ann Am Thorac Soc. 2014;11(10):1592-1596. doi: 10.1513/AnnalsATS.201406-244BC

18. Salottolo K, Offner PJ, Orlando A, et al. The epidemiology of do-not-resuscitate orders in patients with trauma: a community level one trauma center observational experience. Scand J Trauma Resusc Emerg Med. 2015;23(1):9. doi: 10.1186/s13049-015-0094-2.

19. Albaeni A, Chandra-Strobos N, Vaidya D, Eid SM. Predictors of early care withdrawal following out-of-hospital cardiac arrest. Resuscitation. 2014;85(11):1455-1461. doi: 10.1016/j.resuscitation.2014.08.030.

20. Lissauer ME, Naranjo LS, Kirchoffner J, Scalea TM, Johnson SB. Patient characteristics associated with end-of-life decision making in critically ill surgical patients. J Am Coll Surg. 2011;213(6):766-770. doi: 10.1016/j.jamcollsurg.2011.09.003.

21. Muni S, Engelberg RA, Treece PD, Dotolo D, Curtis JR. The influence of race/ ethnicity and socioeconomic status on end-of-life care in the ICU. Chest. 2011;139(5):1025-1033. doi: 10.1378/chest.10-3011.

22. Rubin MA, Dhar R, Diringer MN. Racial differences in withdrawal of mechanical ventilation do not alter mortality in neurologically injured patients. J Crit Care. 2014;29(1):49-53. doi: 10.1016/j.jcrc.2013.08.023.

23. Mayer SA, Kossoff SB. Withdrawal of life support in the neurological intensive care unit. Neurology. 1999;52(8):1602-1609. doi: 10.1212/WNL.52.8.1602. 
24. 2nd National Congress on Medicinal Plants. Iranian J Pharm Res. 2013;12:43.

25. Hamel MB, Phillips R, Teno J, et al. Cost effectiveness of aggressive care for patients with nontraumatic coma. Crit Care Med. 2002;30(6):1191-1196.

26. Reichner CA, Thompson JA, O'Brien S, Kuru T, Anderson ED. Outcome and code status of lung cancer patients admitted to the medical ICU. Chest. 2006:130(3):719-723. doi: 10.1378/chest.130.3.719.

27. Geocadin RG, Buitrago MM, Torbey MT, Chandra-Strobos N, Williams MA, Kaplan PW. Neurologic prognosis and withdrawal of life support after resuscitation from cardiac arrest. Neurology. 2006;67(1):105-108. doi: 10.1212/01. wnl.0000223335.86166.b4.

28. Weimer JM, Nowacki AS, Frontera JA. Withdrawal of life-sustaining therapy in patients with intracranial hemorrhage: self-fulfilling prophecy or accurate prediction of outcome? Crit Care Med. 2016;44(5):1161-1172. doi: 10.1097/ CCM.0000000000001570.

29. Mulder M, Gibbs HG, Smith SW, et al. Awakening and withdrawal of life-sustaining treatment in cardiac arrest survivors treated with therapeutic hypothermia. Crit Care Med. 2014;42(12):2493-2499. doi: 10.1097/ CCM.0000000000000540.

30. Brown CE, Engelberg RA, Nielsen EL, Curtis JR. Palliative care for patients dying in the intensive care unit with chronic lung disease compared with metastatic cancer. Ann Am Thorac Soc. 2016;13(5):684-689. doi: 10.1513/AnnalsATS.201510-667OC

31. Plaisier BR, Blostein PA, Hurt KJ, Malangoni MA. Withholding/withdrawal of life support in trauma patients: is there an age bias? Am Surg. 2002;68(2):159-162.

32. Beauchamp, Childress JF. Principles of Biomedical Ethics. 13th ed. Oxford: Oxford University Press; 2013

33. Jonson AR, Siegler M, Winslade WJ. Clinical Ethics: A Practical Approach to Ethical Decisions in Clinical Medicine. New York: McGraw Hill; 2015

34. Johnson KS, Elbert Avila KI, Tulsky JA. The influence of spiritual beliefs and practices on the treatment preferences of African Americans: a review of the literature. J Am Geriatr Soc. 2005;53(4):711-719. doi: 10.1111/j.15325415.2005.53224.x.

35. Mark NM, Rayner SG, Lee NJ, Curtis JR. Global variability in withholding and withdrawal of life-sustaining treatment in the intensive care unit: a systematic review. Intensive Care Med. 2015;41(9):1572-1585. doi: 10.1007/s00134-0153810-5.

36. Creutzfeldt CJ, Wunsch H, Curtis JR, Hua M. Prevalence and Outcomes of Patients Meeting Palliative Care Consultation Triggers in Neurological Intensive Care Units. Neurocrit Care. 2015;23:14-21.

37. Mulder M, Smith SW, Dhaliwal RS, Goodwin HE, Scott NL, Geocadin RG. Comatose survivors of cardiac arrest and therapeutic hypothermia: Time of awakening and withdrawal of life sustaining therapies. Neurocrit Care. 2013;19:S281.

38. Naib T, Lahewala S, Arora S, Gidwani U. Palliative care in the cardiac intensive care unit. Am J Cardiol. 2015;115:687-90.

39. Prendergast TJ, Luce JM. Increasing incidence of withholding and withdrawal of life support from the critically ill. Am J Respir Crit Care Med. 1997;155:15-20.

40. Smedira NG, Evans BH, Grais LS, et al. Withholding and withdrawal of life support from the critically ill. N Engl J Med. 1990;322:309-15.

41. Van Scoy LJ, Sherman M. Factors Affecting Code Status in a University Hospital Intensive Care Unit. Death Stud. 2013;37:768-81.

42. White DB, Curtis JR, Lo B, Luce JM. Decisions to limit life-sustaining treatment for critically ill patients who lack both decision-making capacity and surrogate decision-makers. Crit Care Med. 2006;34:2053-9.

43. Kerlin MP, Harhay MO, Kahn JM, Halpern SD. Nighttime intensivist staffing, mortality, and limits on life support; a retrospective cohort study. Chest. 2015;147(4):951-958

44. Kish Wallace S, Martin CG, Shaw AD, Price KJ. Influence of an advance directive on the initiation of life support technology in critically ill cancer patients. Crit Care Med. 2001;29(12):2294-2298. 